\title{
Application of a simple quantum chemical approach to ligand fragment scoring for Trypanosoma brucei pteridine reductase 1 inhibition
}

\author{
Wiktoria Jedwabny $^{1} \cdot$ Joanna Panecka-Hofman $^{2,3} \cdot$ Edyta Dyguda-Kazimierowicz $^{1}(0)$ \\ Rebecca C. Wade ${ }^{2,4,5} \cdot$ W. Andrzej Sokalski ${ }^{1}$
}

Received: 28 September 2016 / Accepted: 16 June 2017 / Published online: 7 July 2017

(C) The Author(s) 2017. This article is an open access publication

\begin{abstract}
There is a need for improved and generally applicable scoring functions for fragment-based approaches to ligand design. Here, we evaluate the performance of a computationally efficient model for inhibitory activity estimation, which is composed only of multipole electrostatic energy and dispersion energy terms that approximate long-range ab initio quantum mechanical interaction energies. We find that computed energies correlate well with inhibitory activity for a compound series with varying substituents targeting two subpockets of the binding site of Trypanosoma brucei pteridine reductase 1. For one subpocket, we find that the model is more predictive for inhibitory activity than the ab initio interaction energy calculated at the MP2 level. Furthermore, the model is found to outperform a commonly used empirical scoring method.
\end{abstract}

Wiktoria Jedwabny and Joanna Panecka-Hofman have contributed equally to the investigation.

Electronic supplementary material The online version of this article (doi:10.1007/s10822-017-0035-4) contains supplementary material, which is available to authorized users.

Edyta Dyguda-Kazimierowicz

Edyta.Dyguda@pwr.edu.pl

1 Department of Chemistry, Wrocław University of Science and Technology, Wrocław, Poland

2 Molecular and Cellular Modeling Group, Heidelberg Institute for Theoretical Studies (HITS), Heidelberg, Germany

3 Centre of New Technologies, University of Warsaw, Warsaw, Poland

4 Center for Molecular Biology (ZMBH), DKFZ-ZMBH Alliance, Heidelberg University, Heidelberg, Germany

5 Interdisciplinary Center for Scientific Computing (IWR), Heidelberg University, Heidelberg, Germany
Finally, we show that the results for the two subpockets can be combined, which suggests that this simple nonempirical scoring function could be applied in fragment-based drug design.

Keywords Pteridine reductase $1 \cdot \mathrm{PTR} 1 \cdot$ Interaction energy $\cdot \mathrm{Ab}$ initio $\cdot$ Nonempirical model $\cdot$ Binding affinity prediction

\section{Introduction}

Empirical computational methods are widely used in ligand discovery projects for ligand docking and computing ligand-receptor binding energetics. Docking procedures using empirical scoring functions are often found to successfully predict poses, but commonly lack sufficient accuracy to correctly rank poses or predict binding affinities [1-4]. On the other hand, rigorous ab initio quantum mechanical methods offer the possibility of more accurate calculations, but are generally too computationally costly and therefore impractical in drug design projects. Nevertheless, quantum chemical calculations may provide deeper insights into the physical nature of the corresponding interactions and lead to simpler and more robust nonempirical models. An example is the finding that for polar or charged inhibitors of phenylalanine ammonialyase and leucine aminopeptidase, the nonempirical first-order electrostatic interaction energy defined within perturbation theory [5] (or its multipole component [6]) alone yielded a reasonable correlation with experimental inhibitory activity data. However, such a simple model is insufficient for nonpolar receptors, like fatty acid amide hydrolase (FAAH), where inclusion of a nonempirical dispersion term, in addition to the electrostatic multipole 
term, was necessary to describe inhibitory activities [7]. Likewise, as noted by Lonsdale et al. [8], dispersion effects should be considered for reliable modeling of enzyme-catalyzed reactions.

While the electrostatic multipole term estimated from atomic multipole moments obtained from RHF wavefunctions scales favorably with the number of atoms $A$ of the studied system as $O\left(A^{2}\right)$, the ab initio calculation of dispersion energy is much more computationally demanding, scaling as $O\left(N^{5}\right)$, where $N$ is the size of the basis set and, as such, it cannot be part of a generally applicable scoring method. A computationally inexpensive empirical expression for the dispersion energy employed by classical force fields [9] might be seen as a rational substitute for the ab initio calculations $[10,11]$. However, empirical dispersion appears to be associated with a non-systematic error compared to rigorous DFT-SAPT results [10]. Another drawback of the classical term seems to arise for intermonomer distances shorter than equilibrium separation, wherein empirical results deviate from the reference DFT-SAPT calculations [11]. Since such shortened intermolecular distances might result from force field inadequacy [12] or basis set superposition error [13], any method including short range intermolecular energy terms sensitive to artificial compression of intermonomer separation is inadequate for the purpose of rapid estimation of the binding energy within protein-ligand complexes.

Most attempts to derive affordable and reliable dispersion corrections have been undertaken in conjunction with density functional theory methods, which do not account for the dispersive van der Waals forces unless special corrections are added [14-16]. Pernal et al. [17] proposed an alternative approach-a dispersion function $E_{D a s}$ that describes noncovalent interactions by atom-atom potentials fitted to reproduce the results of high-level SAPT (Symmetry Adapted Perturbation Theory [18]) calculations that provide state-of-the-art quantum chemical dispersion and exchange-dispersion energies. It is noteworthy that the $E_{\text {Das }}$ function demonstrated remarkable performance in describing hydrogen bonding interactions, which are governed by both electrostatic and dispersive forces [19]. The low computational cost of this approximate dispersion function and its broad applicability stemming from the lack of empirical parametrization, make the use of the $E_{\text {Das }}$ expression a promising approach to describing dispersive contributions in scoring methods suited for virtual screening. Further advantages of the $E_{\text {Das }}$ term over van der Waals $1 / \mathrm{r}^{6}$ empirical expression discussed above are the clear physical meaning of the former and its pertinence to a wide range of intermolecular distances because of an additional higher order $1 / \mathrm{r}^{8}$ term and an exponential damping function that is essential at short distances where penetration effects become significant.
Here, we evaluate the ability of the simple model that was previously tested for a congeneric series of inhibitors of the FAAH protein [7], to predict the activities of inhibitors targeting two different subpockets of a protein binding site, which is an important requirement for application in fragment-based drug design approaches. In this model, the ligand-receptor interaction energy is approximated by the sum of the first-order electrostatic multipole component of the interaction energy, $E_{E L, M T P}^{(10)}$, and $E_{D a s}$, the aforementioned approximation of dispersion energy. The advantage of such a model is that it captures both long-range electrostatic and dispersive energy terms, while being relatively computationally efficient. The interaction energy of an inhibitor or a fragment of an inhibitor with the protein binding pocket is computed in a pairwise manner as the sum of amino-acid residue/inhibitor interaction energies for a set of residues defining the pocket or subpocket.

To validate the $E_{E L, M T P}^{(10)}+E_{D a s}$ approximation, here we compute several contributions to the second-order Møller-Plesset (MP2) interaction energy and assess their importance by evaluating correlation coefficients with experimentally determined inhibitory activities [20]. In these inhibitory activity models, we neglect the influence of binding free energy contributions such as entropy, desolvation energy and conformational adaptation of ligands and receptor upon binding. Our results suggest that this is a valid approximation when considering the relative binding free energies of a congeneric series of inhibitors that are expected to have similar binding modes. In addition, we examine various nonempirical representations of the dispersion term, to test the validity of the $E_{D a s}$ approximation and the possibility of exchanging $E_{\text {Das }}$ with other dispersion corrections used with various DFT functionals. It should be noted that such corrections represent not only dispersion interactions but also other nonphysical deficiencies of DFT functionals [17].

In this study, we perform calculations for pteridine reductase 1 (PTR1), an enzyme involved in the pterin metabolism of trypanosomatid parasites [21, 22]. This enzyme, which is present in parasites but not humans, is a target for the design of inhibitors [20, 23-25] that disrupt the reduction of biopterin and folate in parasites and thus hinder their growth. In particular, PTR1 is an important enzyme in Trypanosoma brucei $(\mathrm{Tb})$, which causes human African trypanosomiasis [26]. Different subpockets in the main binding site of TbPTR1 have been explored in inhibitor discovery projects and it therefore provides a good system for assessing the applicability of the simple nonempirical model for fragment-based approaches to inhibitor design.

In this work, we focus on the benzimidazol-2-amine series of potent, non-covalent, and reversible TbPTR1 inhibitors that was developed by Mpamhanga et al. [23] 


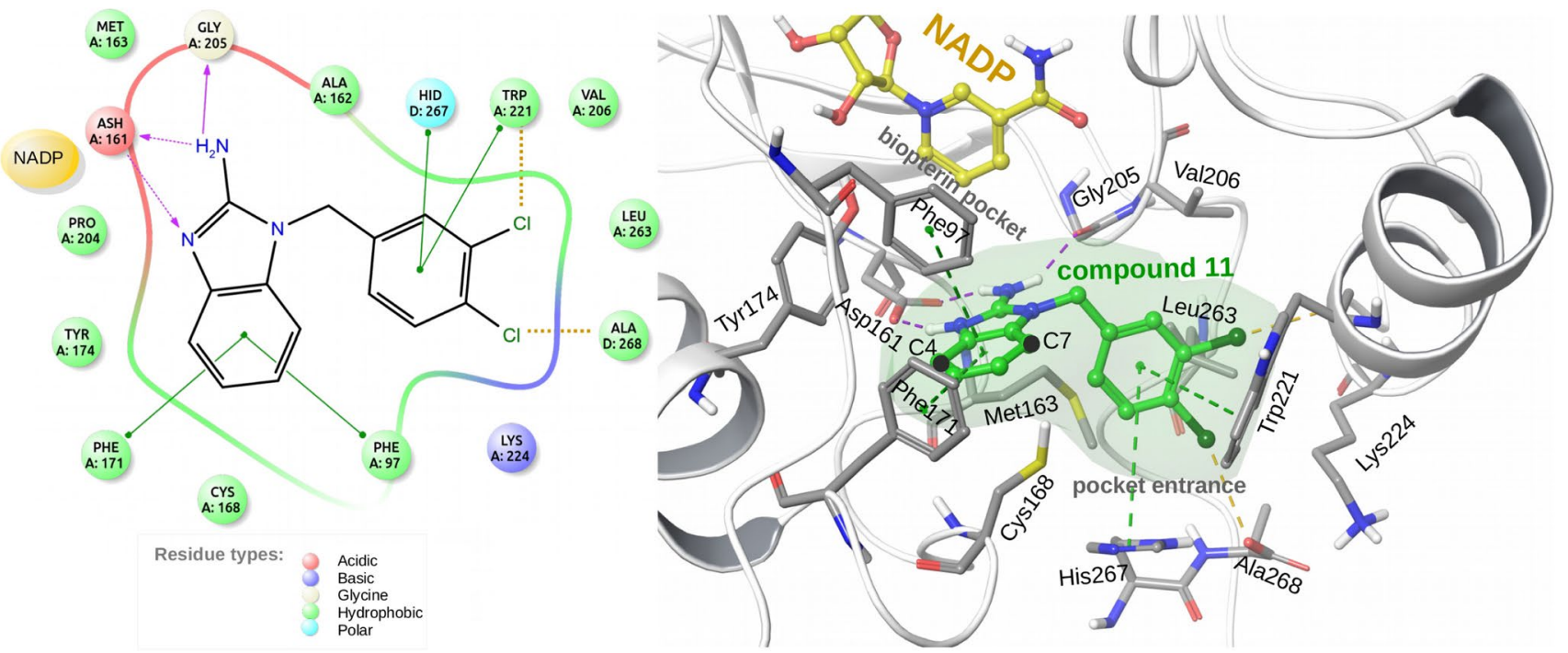

Fig. 1 The crystal structure of TbPTR1 in complex with compound 11 (PDB: 3GN2) showing the interactions made by the inhibitor in the binding site. (left) Interaction diagram of compound 11 with the TbPTR1 protein pocket. A and D denote protein subunits in the TbPTR1 homotetramer. (right) View of compound 11 (green with green semi-transparent surface contour) in the TbPTR 1 binding site, with residues within $3 \AA$ of the ligand shown in stick represen- tation and labeled. A fragment of the cofactor, nicotinamide adenine dinucleotide phosphate (NADP), is shown in yellow. The protein is rendered in cartoon representation. The substitution points $(\mathrm{C} 4$ and C7) in compound 11 are labeled. The edge-face $\pi-\pi$ interactions between the inhibitor and the protein are indicated by green dashed lines, while purple and yellow dashed lines denote hydrogen bonds and halogen bonds, respectively

In particular, the $\mathrm{N} 3$ nitrogen of benzimidazole and the 2-amino group make hydrogen-bonds with the carboxylate group of the nearby Asp161 and the backbone carbonyl of Gly205 residue. On the other side of the binding pocket, the chlorines of the 3,4-dichlorophenyl moiety make halogen bonds with the backbone carbonyl group of $\operatorname{Trp} 221$ and the carboxylate group of the terminal Ala268 residue. The position of the scaffold of compound 11 is additionally stabilized by edge-face $\pi-\pi$ interactions (Fig. 1 ). Due to this extensive interaction pattern, we expect similar binding modes for the derivatives of compound 11 . This assumption

Fig. 2 Chemical structures of the $C 7$ set of $T b$ PTR 1 inhibitors [20]. The $\mathrm{C} 7$ position of the benzimidazole moiety is marked in yellow<smiles>Nc1nc2ccccc2n1Cc1ccc(Cl)c(Cl)c1</smiles><smiles>Nc1nc2cccc(Cl)c2n1Cc1ccc(Cl)c(Cl)c1</smiles><smiles>CCCOc1cccc2nc(N)n(Cc3ccc(Cl)c(Cl)c3)c12</smiles><smiles>Nc1nc2cccc(OCc3ccccc3)c2n1Cc1ccc(Cl)c(Cl)c1</smiles><smiles>Nc1nc2cccc(-c3ccccc3)c2n1Cc1ccc(Cl)c(Cl)c1</smiles><smiles>COc1cccc2nc(N)n(Cc3ccc(Cl)c(Cl)c3)c12</smiles> 
was used to model the TbPTR1-inhibitor complexes, for which crystallographic structures were not available.

To evaluate the $E_{E L, M T P}^{(10)}+E_{\text {Das }}$ model for prediction of inhibitory activity, we first analyzed TbPTR1 derivatives of compound 11 substituted at $\mathrm{C} 7$ of the benzimidazole scaffold, i.e. the compounds reported in Fig. 2 (which we refer to as the $C 7$ set). A similar analysis was previously performed for the docked covalent inhibitors of the FAAH enzyme [7]. The FAAH inhibitors were however modelled without knowledge of the crystallographically confirmed binding mode of the core scaffold, which probably introduced uncertainty into the results of the scoring model. Here, our first aim was therefore to test the performance of the $E_{E L, M T P}^{(10)}+E_{D a s}$ model for another protein target, $T b P T R 1$, with an inhibitor series with a well-defined binding mode. Our second aim was to investigate whether the model is general and additive, which is assumed since the interaction energy is calculated as a sum of the pairwise residue/inhibitor contributions. Thus, we made a similar model based on the model for the $C 7$ set for the dataset of the C4-substituted compounds shown in Fig. 3 (which we refer to as the $C 4$ set), with substituents interacting predominantly with a different set of residues than the $C 7$ set. Our models for the $C 4$ and $C 7$ sets show transferability of the $E_{E L, M T P}^{(10)}+E_{D a s}$ model, suggesting its applicability to fragment-based drug design approaches.
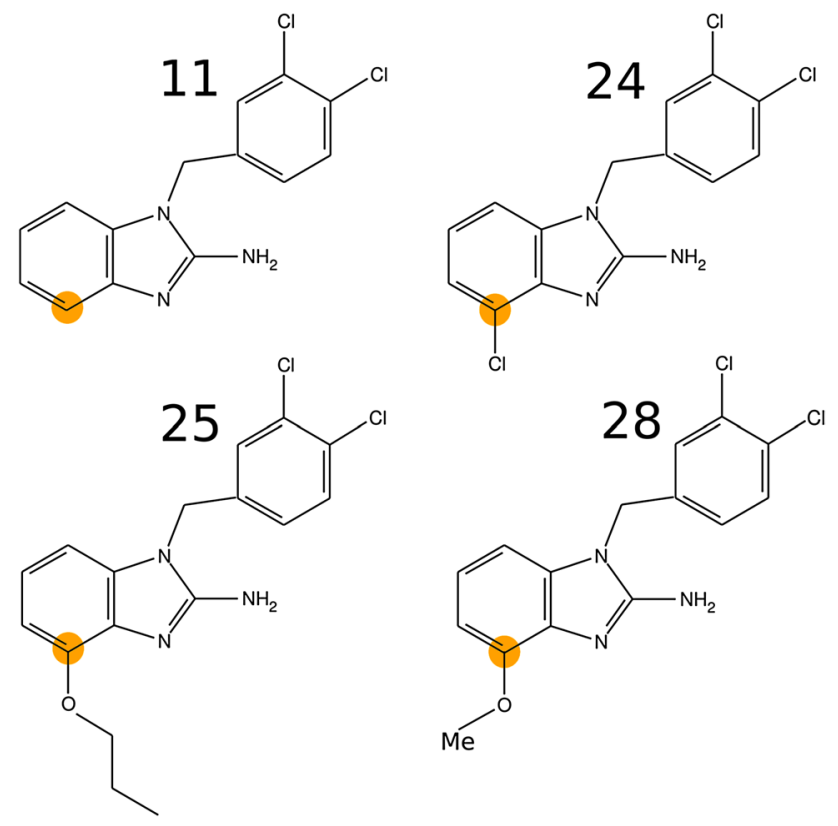

Fig. 3 Chemical structures of the $C 4$ set of TbPTR1 inhibitors [20]. The $\mathrm{C} 4$ position of the benzimidazole moiety is marked in orange

\section{Methods}

\section{Preparation of the sets of compounds}

Calculations were performed for $(i)$ inhibitors from the $C 7$ set [20] (Fig. 2) and (ii) inhibitors from the $C 4$ set [20] (Fig. 3), which share a common parent scaffold: compound 11. The numbering of the inhibitors is adopted from Spinks et al. [20]. Of the reported inhibitors with substitutions at the C4 carbon of benzimidazole [20], two compounds, 26 and 27, were not included in the $C 4$ set due to their weak inhibitory activities and because their binding modes presumably differ from that characterizing the remaining inhibitors (as suggested by our docking simulations). Further assumptions underlying our approach, i.e., the representation of the receptor and ligand structures with models of limited size, preclude consideration of inhibitors with entirely different binding poses. Compound 28 (Fig. 3) was reported to be contaminated with $25 \%$ of compound 33 (Fig. 2), and the inhibitory activity was measured for a mixture [20]. Thus, we calculated the inhibitory activity of the pure compound 28 based on the equilibrium equations for competitive binding. As reported in the Supplementary material, Section 1 , the computed value was $K_{i}^{a p p}=0.42 \mu \mathrm{M}$.

\section{Modeling of the protein-inhibitor complexes}

The binding poses of the C7-set inhibitors were modelled in the TbPTR1 binding pocket based on the crystallographic binding mode of compound 11 (PDB code $3 \mathrm{GN} 2$ [23]). Following the recommended protein preparation protocol [27], crystallographic water molecules were removed and the resulting protein-inhibitor complexes were minimized in Maestro [28] using Protein Preparation Wizard [29] with the default OPLS 2005 [30] force field, and the convergence criterion defined as the non-hydrogen atom RMSD $=0.3 \AA$. Optimal hydrogen bonding was determined with PROPKA [31-34], implemented in Maestro, at $\mathrm{pH} 6.0$, i.e. the $\mathrm{pH}$ used for the measurements of inhibitory activities [20].

The modelling procedure was similar for the $\mathrm{C} 4-$ and $\mathrm{C} 7-$ substituted compounds. However, for the $C 4$ set, the modelling was more difficult because the TbPTR1 subpocket accommodating the $\mathrm{C} 4$ benzimidazole substituents is smaller and more enclosed than that for the $C 7$ set. The binding pose of inhibitor 24 of the $C 4$ set was modelled as described above, but we also analyzed the unminimized protein-inhibitor complex. For compounds 25 and 28, the conformations of the top score poses from QM-polarized docking (implemented in Maestro software suite [28]) were taken for the aforementioned minimization and structure preparation protocol. The QM-polarized 
docking procedure consisted of the following steps: $(i)$ Glide docking with XP (extra-precision) score $[35,36]$ and standard molecular mechanics OPLS 2005 force field electrostatic charges, (ii) recalculation of the semi-empirical Coulson's electrostatic point charges for the docked ligands in the protein surrounding, and (iii) redocking of the inhibitors with the electrostatic charges calculated in step (ii).

\section{Definition of the ligand fragments and TbPTR1 binding subpockets}

Since the binding mode of the common core of the inhibitors considered (i.e., compound 11) is well defined and likely to be positioned similarly for all inhibitors, its contribution to the observed binding affinity differences is most probably negligible. Therefore, to decrease the computational cost for the ab initio interaction energy calculations, the inhibitor structures were truncated to the fragments shown in Fig. 4 (named with a 'fr-' prefix). All the calculations refer to the inhibitor fragments shown in Fig. 4.

The TbPTR1 C7 binding subpocket consisted of residues from the first shell surrounding the $C 7$ inhibitor fragments, see Fig. 5. Due to the potential flexibility of Cys168 and the limited ability of the restrained optimization protocol to account for more extensive conformational changes, Cys168 was excluded from the subpocket definition. Finally, the following residues were present in the $C 7$ receptor model used for evaluating the interaction energy: Phe97, Phe171, Pro210, Met213, Glu217 and Trp221. Since Glu217 was hydrogen-bonded to Trp221, this residue was included in the calculations as a part of a Glu217-Trp221 dimer. The broken bonds arising from cutting the residues out of the protein scaffold were capped with hydrogen atoms optimized with Maestro using the protocol described above. The $C 4$ system consisted of the following residues: Phe97, Asp161, Met163, Val164,

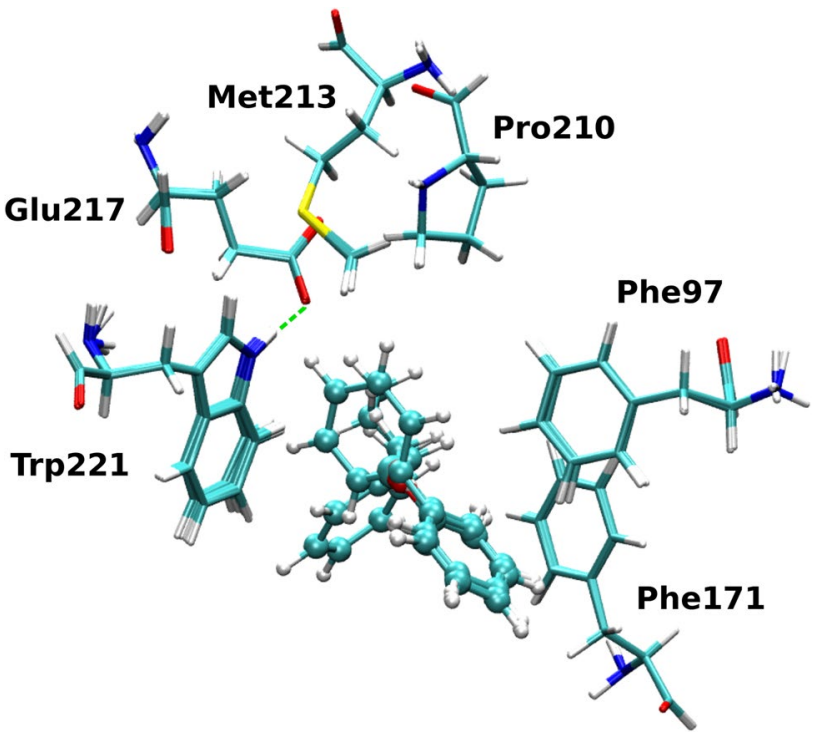

Fig. 5 The $C 7$ subpocket and ligand fragments. Superimposed structures of the complexes of TbPTR1 with the $C 7$ inhibitor fragments showing the surrounding residues included in the calculations. The hydrogen bond between Glu217 and Trp221 is shown by a green dashed line

Pro167, Cys168, Phe171, Tyr174, and Asn175, see Fig. 6. In this subsystem, in contrast to $C 7$, Cys 168 was included, because the rigid backbone of Cys is relatively close to the C4 substitution site, whereas the flexible side chain is not in direct contact (sulphur atom of Cys168 is located about $4.05 \AA$ from carbon C4). Furthermore, in the main set of results, Asp161 was treated as protonated and the protonation of Asp161 at acidic pH is consistent with the catalytic mechanism of PTR1, as suggested by Gourley et al. [37]. Notably, the systems with protonated Asp161 display significantly better correlation of the energy contributions with the $p \mathrm{~K}_{\mathrm{i}}^{a p p}$ values than those with unprotonated Asp161<smiles>CCCOc1ccccc1OCc1ccccc1OCc1ccccc1OCc1ccc(F)cc1</smiles>

Fig. 4 Fragments of the TbPTR1 inhibitors defined for calculations. Carbon atoms corresponding to the C7 and C4 positions of benzimidazole in the full inhibitor structures are marked in yellow and orange, respectively 


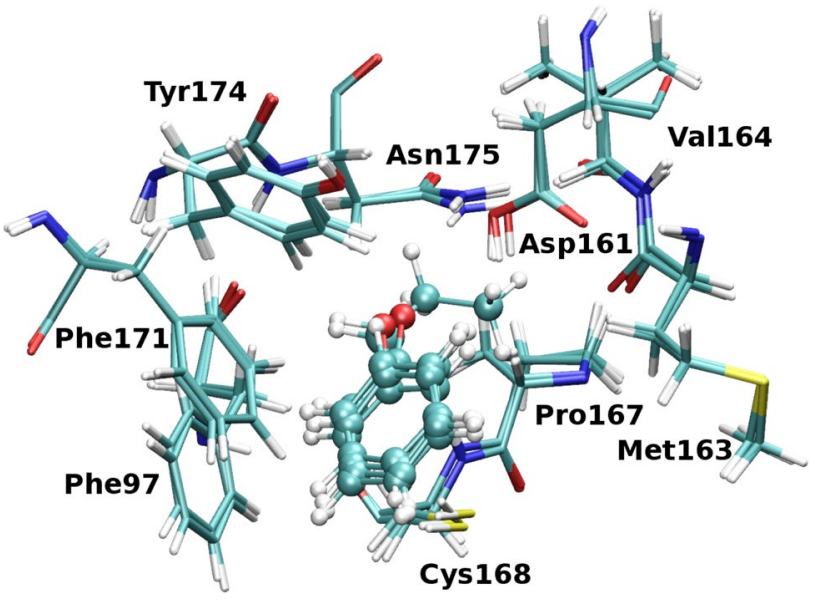

Fig. 6 The $C 4$ subpocket and ligand fragments. Superimposed structures of the complexes of TbPTR1 with the $C 4$ inhibitor fragments showing the surrounding residues included in the calculations

(data shown in Tables S5 and S6 in the Supplementary Material).

\section{Ab initio energy decomposition scheme}

Hybrid variation-perturbation theory (HVPT) [38, 39] was applied to partition the interaction energy calculated at the Møller-Plesset second-order level of theory, $E_{M P 2}$, into the following contributions characterized by increasing computational cost, as indicated by $O(X)$ scaling (where $N$ and $A$ stand for the number of atomic orbitals and atoms, respectively):

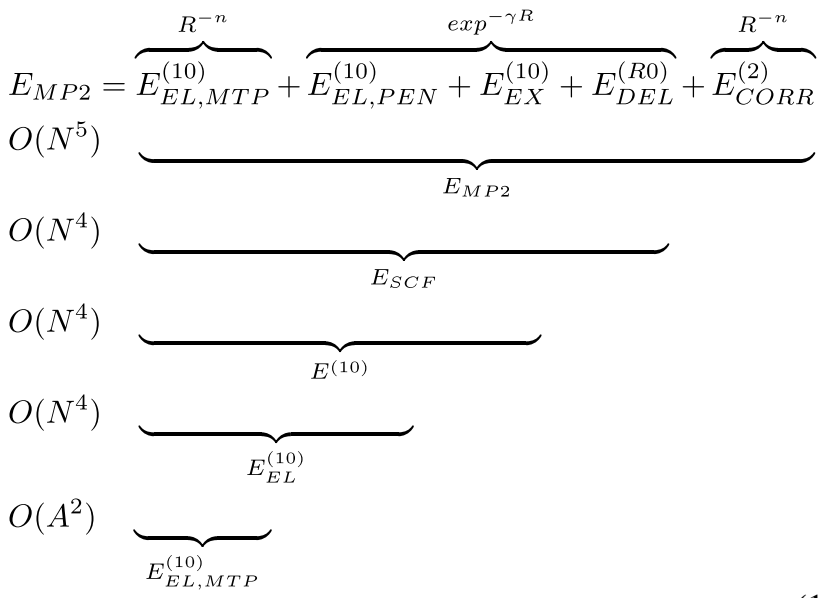

$E_{E L, M T P}^{(10)}$ refers to the electrostatic multipole component estimated from an atomic multipole expansion [40] (see the Supplementary Material for a detailed description of the interaction energy terms discussed herein). $E_{E L, P E N}^{(10)}$ is the electrostatic penetration energy, calculated from the following expression: $E_{E L, P E N}^{(10)}=E_{E L}^{(10)}-E_{E L, M T P}^{(10)}$, where $E_{E L}^{(10)}$ represents the first-order electrostatic energy. The firstorder exchange energy $E_{E X}^{(10)}$ term in Eq. 1 is calculated from the first-order Heitler-London energy, $E^{(10)}: E_{E X}^{(10)}=E^{(10)}$ - $E_{E L}^{(10)}$. The higher-order delocalization energy, $E_{D E L}^{(R 0)}$, is calculated as: $E_{D E L}^{(R 0)}=E_{S C F}-E^{(10)}$, where $E_{S C F}$ is the counterpoise-corrected self-consistent field variational energy. The correlation term is defined as: $E_{C O R R}^{(2)}=E_{M P 2}-E_{S C F}$. The $E_{E L, M T P}^{(10)}$ and $E_{C O R R}^{(2)}$ contributions are considered longrange energy terms, i.e. they vary with the intermolecular distance $R$ as $R^{-n}(n \in \mathbb{N})$, whereas $E_{E L, P E N}^{(10)}, E_{E X}^{(10)}$ and $E_{D E L}^{(R 0)}$ are short-range terms, i.e. they vary as $\exp ^{-\gamma R}$ with $R, \gamma>0$ (see Eq. 1).

\section{Ab initio interaction energy calculations}

The interaction energy between each residue (a single residue monomer or the Glu217-Trp221/Met163-Val164 dimers) and each inhibitor fragment was calculated with a modified version [39] of the GAMESS program [41] using the 6-311G(d) [42, 43] basis set with diffuse functions on the $\mathrm{s}$ and $\mathrm{p}$ orbitals of the chlorine atoms [44, 45]. This approach was chosen rather than using a full $6-311++\mathrm{G}(2 \mathrm{~d}, 2 \mathrm{p})$ basis set to save computational time and because we did not notice major qualitative differences in the results (a comparison is given in the Supplementary material, Table S1). A counterpoise correction was applied to avoid basis set superposition error [46].

Multipole electrostatic energy terms were calculated using the Cumulative Atomic Multipole Moments (CAMM) approach [40, 47] implemented in GAMESS with the expansion truncated at the $R^{-3}$ term. Exponential truncation of the CAMM expansion at the $(1 / R)^{n}$ term seems to limit the disruptive effect of diffuse functions on higher rank multipoles and yields the best results for $n=3$, i.e. including the atomic dipole-dipole and monopole-quadrupole terms.

In addition, three dispersion energy models were tested and compared as regards their suitability for this application, namely: $(i)$ as a reference, the $E_{C O R R}^{(2)}$ term from the HVPT energy decomposition scheme, (ii) the $E_{\text {Das }}$ function [17, 19] fitted to the SAPT [18] results, and (iii) the D3 correction [48] to dispersionless-DFT with Becke-Johnson damping [49] applied. The latter was calculated with standalone DFT-D3 program (version 3.1. Rev 1) [48, 49], using three different functionals, namely the hybrid functionals PBE0 [50, 51] and B3LYP [52], and the exchange functional TPSS [53]. D3 is defined as a dispersion correction being the sum of two- and three-body contributions to the dispersion energy [48]. 


\section{Data analysis}

Each scoring model considered was compared with the experimental inhibitory activities $\left(p \mathrm{~K}_{\mathrm{i}}^{a p p}\right)$ by computing Pearson correlation coefficients $(\mathrm{R})$ and the success rate of prediction of relative binding affinities $\left(N_{\text {pred }}\right)$. The latter is defined as the percentage of concordant inhibitor pairs with relative stability of the same sign as in the reference experimentally measured activities, evaluated among all pairs of the inhibitor set ( $C 4$ or $C 7$ ) [54]. Note that when Pearson correlation coefficients between the calculated interaction energies in our model and the experimental inhibitory activities are negative (trends of the aforementioned data sets are opposite), these values are said to correlate, as lower values of energy should ideally accompany increasing inhibitory activity. For the inverse relationship associated with a positive Pearson correlation coefficient, we use the term "anticorrelation". The XP (extra-precision) scoring in the Glide program [35, 36, 55], with the "scorein-place" option, was applied for empirical scoring of the fragments used for the ab initio calculations. The score function is expressed as follows:

$X P$ GlideScore $=E_{\text {coul }}+E_{v d W}+E_{\text {bind }}+E_{\text {penalty }}$

$E_{c o u l}$ and $E_{v d W}$ are electrostatic and van der Waals terms, respectively, $E_{\text {penalty }}$ includes a desolvation penalty and a ligand strain energy which are unfavorable for binding, while $E_{\text {bind }}$ is composed of favourable terms similar to those in the ChemScore function [56]. The $E_{\text {bind }}$ terms of the XP GlideScore additionally include: $(i)$ a model of hydrophobic interactions, which takes into account ligand hydrophobic enclosure, and (ii) an improved model of hydrogen bond interactions [36]. The above scoring function was tested on 198 protein-ligand complexes, resulting in binding free energy RMSDs of 2.26 and $1.73 \mathrm{kcal} \mathrm{mol}^{-1}$ over all and selected well-docked ligands, respectively, as reported by Friesner et al. [36].

\section{Results and discussion}

\section{The $E_{E L}^{(10)}$ and $E_{M P 2}$ interaction energies correlate with $p \mathrm{~K}_{i}^{a p p}$ values of the $C 7$ set}

The total interaction energy values calculated at the consecutive levels of theory are shown in Table 1. Moreover, the relationship between these interaction energies and exchange, delocalization and correlation contributions is depicted in Fig. S1 in the Supplementary Material. The corresponding pairwise interaction energy values for inhibitor-residue pairs and the numerical values of exchange and delocalization energy components are presented in the Supplementary material (Tables S2, S3, respectively).

Table 1 shows that the $E_{M P 2}$ interaction energy significantly correlates with the experimentally determined inhibitory activities $(\mathrm{R}=-0.89)$. Apart from fr-29, the $E_{M P 2}$ values reflect the ranking of the inhibitors established experimentally. Furthermore, the $E_{E L}^{(10)}$ values correlate with the experimental data $(\mathrm{R}=-0.84)$, but with a lower $N_{\text {pred }}$ value than for $E_{M P 2}$ ( 80 vs. $86.7 \%$, respectively).

For the fragments of three least potent inhibitors of the $C 7$ set, fr-29, fr-33, and fr- 11 , the differences in $p \mathrm{~K}_{\mathrm{i}}$ of about 0.1 (corresponding to an approximately $0.1 \mathrm{kcal}$ $\mathrm{mol}^{-1}$ difference in binding free energy [57]), cannot be expected to be reproduced computationally, as they exceed the accuracy of most quantum chemical calculations, and, likely, of the experiments (measurement errors are, however, not explicitly provided in Refs. [20, 23]). Accordingly,
Table 1 The total interaction energy at consecutive levels of theory, and energies for the $E_{E L, M T P}^{(10)}+E_{D a s}$ and $E_{E L, M T P}^{(10)}+E_{C O R R}^{(2)}$ models for the $C 7$ set of inhibitors

\begin{tabular}{lllllllll}
\hline Inhibitor & $p \mathrm{~K}_{\mathrm{i}}^{a p p} \mathrm{a}$ & $E_{E L, M T P}^{(10)}$ & $E_{E L}^{(10)}$ & $E^{(10)}$ & $E_{S C F}$ & $E_{M P 2}$ & $E_{E L, M T P}^{(10)}+E_{\text {Das }}$ & $E_{E L, M T P}^{(10)}+E_{C O R R}^{(2)}$ \\
\hline $\mathrm{fr}-32$ & 8.2 & -4.4 & -8.3 & 10.8 & 7.0 & -9.5 & -25.8 & -20.9 \\
$\mathrm{fr}-30$ & 7.3 & -3.4 & -6.9 & 7.6 & 4.5 & -8.8 & -21.9 & -16.7 \\
$\mathrm{fr}-31$ & 7.0 & -0.2 & -7.0 & 12.2 & 8.9 & -7.9 & -20.2 & -16.9 \\
$\mathrm{fr}-29$ & 6.3 & -1.3 & -3.0 & 6.2 & 4.6 & -4.0 & -12.2 & -9.8 \\
$\mathrm{fr}-33$ & 6.2 & -3.0 & -5.9 & 5.3 & 3.3 & -6.4 & -15.5 & -12.7 \\
$\mathrm{fr}-11$ & 6.1 & -2.5 & -4.6 & 5.1 & 3.6 & -4.1 & -12.4 & -10.1 \\
$\mathrm{R}^{\mathrm{b}}$ & & -0.48 & -0.84 & 0.76 & 0.62 & -0.89 & -0.96 & -0.95 \\
$N_{\text {pred }}^{\mathrm{c}}$ & & 66.7 & 80.0 & 13.3 & 26.7 & 86.7 & 86.7 & 80.0 \\
\hline
\end{tabular}

In units of kcal mol${ }^{-1}$

${ }^{\mathrm{a}}$ Experimental $p \mathrm{~K}_{\mathrm{i}}^{a p p}$ values are taken from Ref. [20]

${ }^{b}$ Pearson correlation coefficient between the calculated energy and the experimental inhibitory activity

${ }^{c}$ Percentage of concordant pairs (\%) 
the computed binding energies of fr- 29 , fr-33, and fr- 11 ligands would be expected to be similar. It can be seen in Table 1 that fr-29 and fr-11, but not fr-33, have similar $E_{M P 2}$ interaction energy values. Actually, the $E_{M P 2}$ binding energies of the $C 7$ set inhibitors with an oxygen atom at the $\mathrm{C} 7$ position (i.e., compounds 30, 31, and 33) appear to be slightly overestimated (see Fig. S2 in the Supplementary material) whereas, the inhibitors with a hydrophobic C7 substituent have underestimated values of $E_{M P 2}$ interaction energy. Notably, the C7 substituents are in direct contact with the methylene linker of the common scaffold depicted in Fig. 2 (e.g., the distance between the $\mathrm{C} 7$ chlorine and the closest hydrogen atom of the adjacent methyl group in compound 29 is $2.7 \AA$ ). Considering that this particular part of the inhibitor was not included in binding energy analysis, the observed under- and overestimation of interaction energy could be associated with intramolecular interactions that are not accounted for by the model. Despite these omissions, the overall correlation with experimental inhibitory activity is satisfactory.

On the other hand, both $E^{(10)}$ and $E_{S C F}$ anti-correlate with inhibitory activities, i.e., these energies tend to indicate greater repulsion for the compounds with higher inhibitory activity. Positive values of the $E^{(10)}$ and $E_{S C F}$ energies result from the repulsive exchange contribution, $E_{E X}^{(10)}$ (Table S3 in the Supplementary material). The repulsion is stronger for more potent inhibitors, since the molecular fragments that interact strongly are also more likely to experience stronger exchange effects $[54,58]$. For example, for fr-31, the $E^{(10)}$ and $E_{S C F}$ interaction energy terms appear to be exceptionally high (i.e., unfavorable) due to the incorporation of short-range exchange contribution. The short-range effects are exponentially dependent on the interatomic distance, so any minor structural defect (e.g., artificial shortening of the intermolecular distance due to the basis set superposition error or the difference between the crystal structure and the gas phase or the force field non-transferability) results in significant errors in the energy values. Therefore, $E^{(10)}$ and $E_{S C F}$ are vulnerable to structural deficiencies in the model $[7,58]$, and are not really applicable for studying crystal structure-based models of the protein-ligand complexes.

Although the delocalization term, $E_{D E L}^{(R 0)}$, accounted for at the Hartree-Fock level of theory is characterized by a correlation coefficient $\mathrm{R}=-0.94$ (see the Supplementary material, Table S3), it is still insufficient to overcome the inverse inhibitory ranking due to the $E_{E X}^{(10)}$ contribution (Fig. S1 in the Supplementary material). Only the $E_{C O R R}^{(2)}$ contribution to the $E_{M P 2}$ energy restores the proper ranking of the inhibitory activities (Table 1). As demonstrated in Fig. S1 in the Supplementary material, both the $E_{D E L}^{(R 0)}$ and $E_{C O R R}^{(2)}$ terms approximately cancel out the repulsive characteristics of the exchange component, but $E_{C O R R}^{(2)}$ is the major contribution out of these two.

The above analysis is in line with our previous findings for FAAH inhibitors [7] that omitting the contributions arising from short-range interactions (i.e., considering only the multipole electrostatic and correlation energy components) results in a much better model for predicting the inhibitory activity [7].

\section{The $E_{D a s}$ approximation predicts inhibitory activities of the $C 7$-set inhibitors as well as the correlation energy}

The results shown in Table 2 indicate that, for the $C 7$-set inhibitors, the dominant dispersion contribution could even serve as a standalone scoring method, with $\mathrm{R}=-0.86$ for $E_{C O R R^{2}}^{(2)}$ Furthermore, for the $C 7$ inhibitor set, the $E_{D a s}$
Table $2 E_{D a s}$ function and the D3 dispersion energy approximations evaluated for the $C 7$-set inhibitor fragments

\begin{tabular}{llclcrr}
\hline Inhibitor & $p \mathrm{~K}_{\mathrm{i}}^{a p p} \mathrm{a}$ & $E_{\text {CORR }}^{(2)}$ & $E_{\text {Das }}$ & $\mathrm{D}_{\text {PBEO }}$ & $\mathrm{D}_{\text {B3LYP }}$ & $\mathrm{D}_{\text {TPSS }}$ \\
\hline $\mathrm{fr}-32$ & 8.2 & -16.5 & -21.4 & -12.3 & -17.6 & -15.7 \\
$\mathrm{fr}-30$ & 7.3 & -13.3 & -18.5 & -10.9 & -15.5 & -13.8 \\
$\mathrm{fr}-31$ & 7.0 & -16.8 & -20.1 & -12.2 & -17.1 & -15.4 \\
$\mathrm{fr}-29$ & 6.3 & -8.5 & -10.9 & -6.5 & -9.2 & -8.2 \\
$\mathrm{fr}-33$ & 6.2 & -9.7 & -12.5 & -7.5 & -10.5 & -9.4 \\
$\mathrm{fr}-11$ & 6.1 & -7.6 & -9.9 & -5.7 & -8.2 & -7.3 \\
$\mathrm{R}_{\text {CORR }}^{\mathrm{b}}$ & & & 0.98 & 0.99 & 0.99 & 0.99 \\
$\mathrm{R}^{\mathrm{c}}$ & & -0.86 & -0.92 & -0.89 & -0.90 & -0.89 \\
$\mathrm{~N}_{\text {pred }}^{\mathrm{d}}$ & & 66.7 & 86.7 & 86.7 & 86.7 & 86.7 \\
\hline
\end{tabular}

In units of kcal mol ${ }^{-1}$

${ }^{a}$ Experimental affinity values are taken from Ref. [20]

${ }^{\mathrm{b}}$ Pearson correlation coefficient between the dispersion approximation and the correlation energy $E_{C O R R}^{(2)}$ ${ }^{c}$ Pearson correlation coefficient between the calculated dispersion approximation and the experimental inhibitory activity taken from Ref. [20]

${ }^{\mathrm{d}}$ Percentage of concordant pairs $(\%)$ 
approximation correlates with the inhibitory activities as well as $E_{C O R R}^{(2)}(\mathrm{R}=-0.92)$, thereby confirming the validity of the $E_{D a s}$ approximation. A significant correlation is obtained for the $E_{C O R R}^{(2)}$ and $E_{D a s}$ energies because the $C 7$-set of inhibitor fragments consists of largely hydrophobic substituents targeting a relatively hydrophobic TbPTR1 subpocket, and thus dispersive interactions play a major role in binding.

Nonetheless, a universal scoring model should also account for the electrostatic contribution, so that it is appropriate for noncovalent complexes with intermolecular forces of either dispersive or electrostatic nature (or a mixture of both). Upon including the electrostatic multipole term, the scoring abilities of the proposed model further improve for the $C 7$ inhibitor set (Table 1): for $E_{E L, M T P}^{(10)}+E_{D a s}$ and $E_{E L, M T P}^{(10)}+E_{C O R R}^{(2)}$ models the correlation coefficients are equal to -0.96 and -0.95 , respectively. Furthermore, the $E_{E L, M T P}^{(10)}+E_{D a s}$ model correctly predicted $86.7 \%$ of concordant pairs, i.e. as many as for the $E_{M P 2}$ level. Note that for the $E_{E L, M T P}^{(10)}+E_{D a s}$ model describing TbPTR1 inhibition, the correlation is distinctly better than for the inhibitors of the FAAH enzyme $(R=-0.67)$ [7]. This difference might be due to the higher flexibility of the FAAH inhibitor series, which may cause problems in determining the correct binding pose even by the most accurate docking procedures.

\section{Comparison of dispersion models: alternatives to the $E_{D a s}$ function}

In the work presented herein, we additionally tested the D3 correction to dispersionless-DFT developed by Grimme et al. [48], calculated for the following functionals: PBE0, B3LYP, and TPSS. Our objective was to check how the simple $E_{\text {Das }}$ approximation is related to the widely tested D3 correction. The performance of the dispersion approximations for the $C 7$ set is compared in Table 2 .

All the dispersion approximations correlate well with the $E_{C O R R}^{(2)}$ results. Considering the agreement with the experimental inhibitory activities, the dispersion energies calculated from the D3 corrections perform comparably well with the other methods, with a correlation coefficient $R$ of about -0.90 for all the three tested functionals, whereas for $E_{D a s}$ and $E_{C O R R}^{(2)}$ the values of $R$ are equal to -0.92 and -0.86 , respectively. While each dispersion approximation considered here performs remarkably well, the dispersion model proposed by Pernal et al. [17] is fitted to the SAPT results, whereas the results obtained with D3 corrections might, in general, depend on the applied functional. Accordingly, the choice of certain functional might not be obvious if structure-activity relationships are to be predicted for novel receptor-ligand complexes

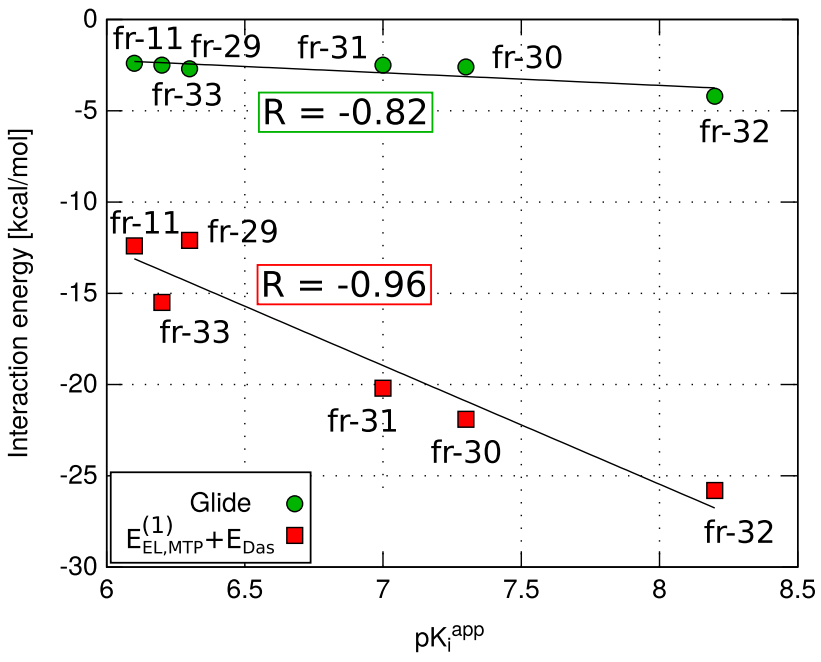

Fig. 7 Interaction energies of the $C 7$ set of TbPTR1 inhibitor fragments computed with nonempirical and empirical scoring approaches versus experimental inhibitory activities taken from Ref. [20]

not characterized experimentally, wherein prior testing of the performance of a given method cannot be carried out.

Comparison of the $E_{E L, M T P}^{(10)}+E_{D a s}$ model with empirical scoring

Protein-ligand interactions are routinely evaluated with computationally inexpensive empirical scoring approaches [59]. These rely on empirical parameters that are typically derived using a diverse training set. Although, in principle, generally applicable, such scoring functions have been shown to perform successfully in some applications and to fail in others [1, 60]. In Fig. 7, the nonempirical $E_{E L, M T P}^{(10)}+E_{D a s}$ scoring method is compared with the Glide XP scoring function [35]; the corresponding values are given in the Supplementary material (Table S4). Notably, the predictive capabilities of the $E_{E L, M T P}^{(10)}+E_{D a s}$ model, tested on the $C 7$ set of inhibitor fragments, outperform the empirical scoring function, with an $R$ correlation coefficient of -0.96 compared to -0.82 value obtained with Glide XP.

An important advantage of the $E_{E L, M T P}^{(10)}+E_{D a s}$ model is that it is fully based on well-defined long-range interaction energy components obtained from first principles ab initio calculations and does not contain any empirical parameters. Moreover, the method is as computationally affordable as commonly used empirical scoring functions, scaling as $O\left(A^{2}\right)$ [7]. Overall, these results suggest that more accurate, nonempirical ways of assessing the protein-ligand interactions might be advantageous for the design of novel inhibitors, and that a gain in the quality of the results can be achieved with little computational effort. 
Table 3 The total interaction energy at consecutive levels of theory, and energies for the $E_{E L, M T P}^{(10)}+E_{D a s}$ and $E_{E L, M T P}^{(10)}+E_{C O R R}^{(2)}$ models for the $C 4$ set of inhibitors

\begin{tabular}{lcccccccc}
\hline Inhibitor & $p \mathrm{~K}_{\mathrm{i}}^{a p p} \mathrm{a}$ & $E_{E L, M T P}^{(10)}$ & $E_{E L}^{(10)}$ & $E^{(10)}$ & $E_{S C F}$ & $E_{M P 2}$ & $E_{E L, M T P}^{(10)}+E_{\text {Das }}$ & $E_{E L, M T P}^{(10)}+E_{C O R R}^{(2)}$ \\
\hline $\mathrm{fr}-25$ & 6.5 & -1.7 & -16.9 & 32.9 & 24.6 & -4.4 & -48.8 & -30.7 \\
$\mathrm{fr}-28$ & 6.4 & -0.1 & -11.7 & 21.4 & 16.0 & -5.8 & -33.4 & -22.0 \\
$\mathrm{fr}-11$ & 6.1 & 1.0 & -9.5 & 19.5 & 14.8 & -2.4 & -22.7 & -16.2 \\
$\mathrm{fr}-24$ & 5.6 & -0.2 & -10.4 & 18.7 & 13.9 & -6.0 & -29.8 & -20.1 \\
$\mathrm{R}^{\mathrm{b}}$ & & -0.44 & -0.66 & 0.70 & 0.71 & 0.21 & -0.59 & -0.59 \\
$N_{\text {pred }}^{c}$ & & 66.7 & 83.3 & 0.0 & 0.0 & 33.3 & 83.3 & 83.3 \\
$\mathrm{fr}^{\mathrm{c}} 4^{*}$ & 5.6 & -2.1 & -17.7 & 27.1 & 19.9 & -1.7 & -36.0 & -23.7 \\
$\mathrm{R}^{* \mathrm{~b}}$ & & 0.27 & 0.28 & 0.14 & 0.17 & -0.85 & -0.35 & -0.33 \\
$N_{\text {pred }}^{*} \mathrm{c}$ & & 50.0 & 50.0 & 33.3 & 33.3 & 83.3 & 66.7 & 66.7 \\
\hline
\end{tabular}

In units of kcal mol${ }^{-1}$

The data for the unminimized compound 24 complex are marked by *

${ }^{a}$ Experimental affinity values are taken from Ref. [20]

bPearson correlation coefficient between the calculated energy and the experimental inhibitory activity

${ }^{\mathrm{c}}$ Percentage of concordant pairs (\%)

\section{Sensitivity of the $E_{E L, M T P}^{(10)}+E_{D a s}$ and $E_{M P 2}$ models \\ to the inhibitor-receptor modelling procedure-the case of compound 24 in the $C 4$ set of inhibitors}

We performed calculations for the $C 4$ set of inhibitor fragments, targeting another subpocket of TbPTR1. Table 3 shows the interaction energies calculated at the different levels of theory, together with the $E_{E L, M T P}^{(10)}+E_{C O R R}^{(2)}$ and $E_{E L, M T P}^{(10)}+E_{\text {Das }}$ models. The corresponding interaction energy values for each residue-inhibitor fragment pair are presented in the Supplementary Material (Table S6).

Since modeling of the $C 4$ set of inhibitor-protein complexes was not as straightforward as for the $C 7$ set, we analyzed the influence of the modeling procedure, in particular the classical force field minimization of the TbPTR1-inhibitor complex, on the computed energy values for one inhibitor, compound 24. In this case, we suspected that the classical force field description [30], which models atoms as spheres with point charges, might incorrectly treat the interactions of 4-Cl substituent with the side chain of Asn175. Due to the complex electronic structure of halogens and the resultant $\sigma$-hole phenomenon, the chlorine may form the so-called halogen bond, which is poorly described in commonly-used force fields [61].

We observed that classical minimization distinctly changes coordinates of this system, with the distance between 4- $\mathrm{Cl}$ of compound 24 and amide $\mathrm{N}$ of Asn175 decreasing from 5.0 to $3.9 \AA$ (see Fig. S3 in the Supplementary material). For the minimized compound 24 complex, the exchange energy $E_{E X}^{(10)}$ is much lower than for the unminimized complex $\left(29.2\right.$ vs. $44.7 \mathrm{kcal} \mathrm{mol}^{-1}$, see Table S7 in the Supplementary material). This difference suggests that minimization reduces some steric clashes in the complex. Furthermore, with minimized compound 24, $E_{E X}^{(10)}$ anti-correlates with $p \mathrm{~K}_{\mathrm{i}}^{a p p}$ for the four $C 4$ compounds, whereas with the unminimized compound 24 complex, no such anti-correlation is observed $(R=0.69$ vs. -0.03 , respectively, Table $\mathrm{S} 7)$. This is likely the reason why $E_{M P 2}$ does not correlate with $p \mathrm{~K}_{\mathrm{i}}^{a p p}$ for the $C 4$ set with minimized inhibitor $24(R=0.21$, Table 3$)$, whereas it correlates for the unminimized compound $24(R=-0.85)$. On the other hand, the $E_{E L, M T P}^{(10)}+E_{D a s}$ model (which does not include $\left.E_{E X}^{(10)}\right)$ shows the expected inverse relation with $p \mathrm{~K}_{\mathrm{i}}^{\text {app }}$ for both the minimized $(R=-0.59)$ and the unminimized ( $R=-0.35$ ) inhibitor 24 (Table 3 ). Thus, the results suggest that the $E_{E L, M T P}^{(10)}+E_{\text {Das }}$ model is more robust than the $E_{M P 2}$-based model to inaccuracies in the models of the inhibitor-receptor complexes.

Limitations of the $E_{E L, M T P}^{(10)}+E_{D a s}$ model due to omission of the exchange energy

For the $C 4$ set, the correlation of $E_{E L, M T P}^{(10)}+E_{D a s}$ with $p \mathrm{~K}_{\mathrm{i}}^{a p p}$ is not as good as for the $C 7$ set (compare Tables 1,3 ). This is likely due to the lower number of inhibitors and the narrow range of $p \mathrm{~K}_{\mathrm{i}}^{a p p}$ values in this set. However, if we investigate how well the $E_{E L, M T P}^{(10)}+E_{D a s}$ model approximates the reference $E_{M P 2}$ energy, omitting compound 25 significantly increases the correlation between $E_{M P 2}$ and $E_{E L, M T P}^{(10)}+E_{D a s}$, which is 0.27 for all inhibitors and 0.93 when compound 25 is excluded from the C4 set (see Table S8 in the Supplementary material). Notably, $E_{E X}^{(10)}$ of compound 25 seems to be overestimated (unfavourable for binding), while $E_{C O R R}^{(2)}\left(\right.$ and $E_{D a s}$ ) are too favourable (see Table S7 in the 
Supplementary material). For this compound, even the standard docking procedure shows that the side chain makes steric clashes, and these contacts could not be properly relaxed by energy minimization procedure (see Fig. S4 in the Supplementary Material). The omission of the $E_{E X}^{(10)}$ energy, and thereby the effects of short-range repulsion, in the $E_{E L, M T P}^{(10)}+E_{D a s}$ model mean that it should not be applied when there are significant ligand-receptor clashes, such as observed for compound 25 . This problem can be detected at the early stage of modeling/docking prior to the QM calculations. Since the $E_{E L, M T P}^{(10)}+E_{D a s}$ model does not properly capture the interactions of inhibitor 25 , we omitted this compound in further analysis.

\section{Putting the $C 7$ and $C 4$ models together}

Analysis of correlations for the $C 4$-set models should be treated with caution since the dataset (excluding inhibitor 25) consists of only three inhibitors with a very narrow $p \mathrm{~K}_{\mathrm{i}}^{a p p}$ range. Therefore, we did not make correlation models for these points alone. Instead, we fitted these data points to the C7-set $E_{E L, M T P}^{(10)}+E_{D a s}$ and $E_{M P 2}$ models, assuming per-residue additivity of the models (see explanation in the Supplementary material), which requires that $\alpha$ parameter in the $E=\alpha \cdot p K_{i}^{a p p}+\beta$ models for the $C 7$ system was kept constant and the $\beta$ parameter was fit by minimizing the deviation of the three $C 4$ experimental $p \mathrm{~K}_{\mathrm{i}}^{a p p}$ values. The use of different $\beta$ values for different subpockets reflects the fact that there is a different energetic contribution to binding for each substituent position but that this contribution can be assumed constant for all compounds with substituents at a given position. Fig. 8 shows the fitted $E_{M P 2}$ and $E_{E L, M T P}^{(10)}+E_{D a s}$ models for the $C 7$ and the $C 4$ set. It can be seen in Fig. 8 that, for both inhibitor sets,

Table 4 Inhibitory activities $p \mathrm{~K}_{\mathrm{i}}^{a p p}$ for the $C 4$ set predicted with the best fitting equations obtained from the $E_{E L, M T P}^{(10)}+E_{D a s}$ and $E_{M P 2}$ models

\begin{tabular}{lllllll}
\hline Inh. & Exp. $^{\mathrm{a}}$ & \multicolumn{2}{l}{$E_{E L, M T P}^{(10)}+E_{\text {Das }}$} & & \multicolumn{2}{l}{$E_{M P 2}$} \\
\cline { 6 - 7 } & & $C 4$ & $C 4^{*}$ & & $C 4$ & $C 4^{*}$ \\
\hline fr-28 & 6.4 & 6.8 & 6.4 & 6.5 & 7.0 \\
$\mathrm{fr}-11$ & 6.1 & 5.1 & 4.8 & 5.1 & 5.7 \\
$\mathrm{fr}-24$ & 5.6 & 6.2 & 6.9 & 6.5 & 5.4 \\
$\mathrm{RMSE}^{b}$ & & 0.7 & 1.0 & & 0.8 & 0.4 \\
\hline
\end{tabular}

The model has the same gradient $\alpha$ as the corresponding $C 7$ model $\left(E=\alpha \cdot p K_{i}^{a p p}+\beta\right)$

The data sets with the unminimized compound 24 complex are marked by *

See model parameters in Table $\mathrm{S} 9$ in the Supplementary material

${ }^{a}$ Experimental $p \mathrm{~K}_{\mathrm{i}}^{a p p}$ values are taken from Ref. [20]

${ }^{\mathrm{b}}$ Root mean square error of $p \mathrm{~K}_{\mathrm{i}}^{a p p}$ prediction
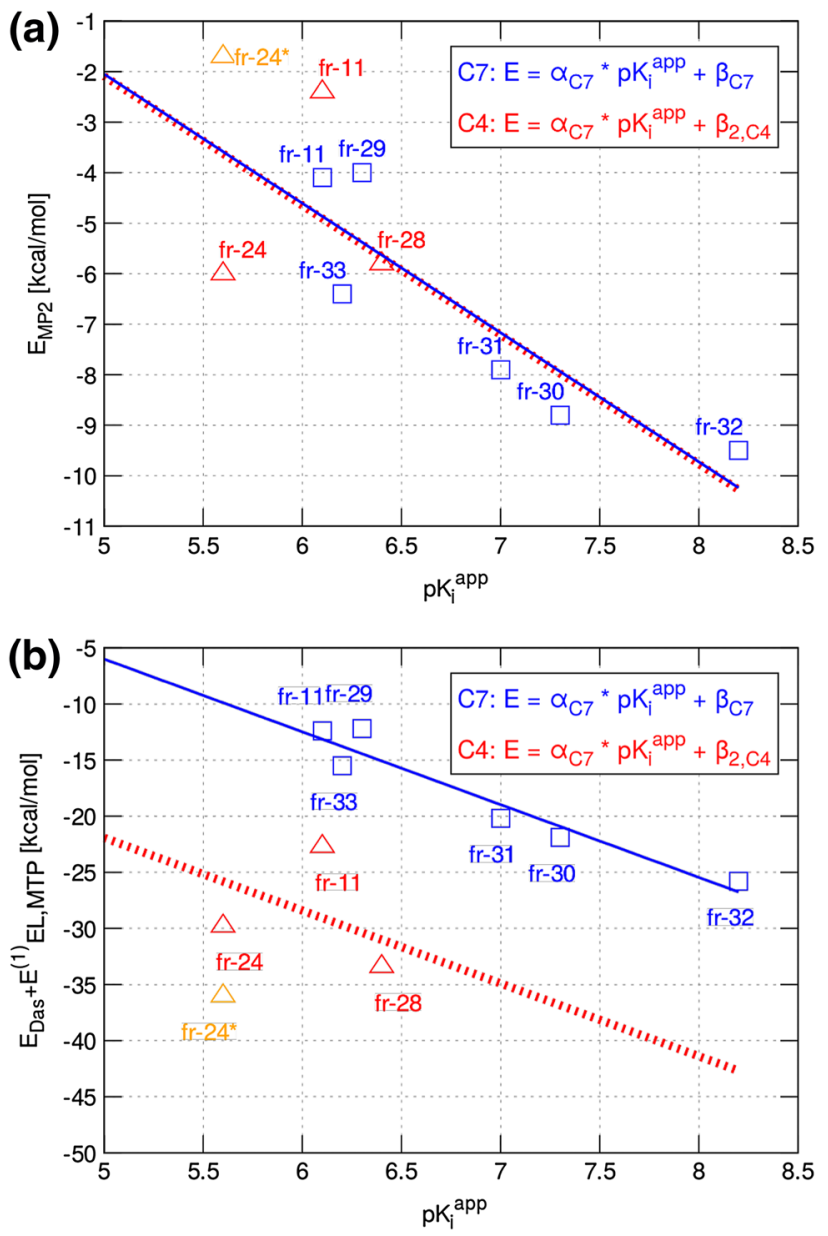

Fig. 8 The $E_{M P 2}(\mathbf{a})$ and $E_{E L, M T P}^{(10)}+E_{D a s}(\mathbf{b})$ interaction energies versus experimental inhibitory activities and linear regression models for the $C 4$ and $C 7$ inhibitor sets. The same $\alpha$ value is used for the $C 7$ and $C 4$ models. Parameters of the models are presented in Table S9 in the Supplementary Material and $p \mathrm{~K}_{\mathrm{i}}^{a p p}$ prediction results are given in Table 4 . The data for the unminimized compound 24 (fr- $\left.24^{*}\right)$ are also shown (in orange)

predictions of the regression models are generally worse for the weaker binders, which form less specific and less stable interactions, and are thus more challenging to model.

The $E_{M P 2}$ models for the $C 7$ set and the $C 4$ or $C 4^{*}$ set (for unminimized inhibitor 24) are remarkably consistent (see Fig. 8a; Table 4). This is confirmed by low RMS errors in $p \mathrm{~K}_{\mathrm{i}}^{a p p}$ values $\left(0.8\right.$ and 0.4 for the $C 4$ and $C 4^{*}$ sets, respectively; Table 4). Thus, the $E_{M P 2}$ model seems to be transferable between the $C 4$ and $C 7$ pockets. The $E_{E L, M T P}^{(10)}+E_{D a s}$ energy performs overall worse in predicting $p \mathrm{~K}_{i}^{a p p}$ values than $E_{M P 2}$ (Table 4), although it also shows reasonable transferability of the $C 7$ model to the $C 4$ subpocket (Fig. 8b; Table 4). The subpockets feature differing physico-chemical properties $(C 7$-more hydrophobic, C4-more hydrophilic; compare Figs. 2, 3), but similar hydrophobic character of the substituents may facilitate the 
transferability of the models between subpockets, which should be further tested for ligands with more diverse properties.

\section{Concluding remarks}

We have conducted ab initio calculations of the interaction energies between a series of inhibitor fragments and binding subpockets of the TbPTR1 enzyme. The common scaffold for this inhibitor series was a derivative of benzimidazol-2-amine (compound 11), with a well-defined binding mode in TbPTR1. Two types of substitutions were analysed, i.e. substituents at the $\mathrm{C} 7$ carbon of benzimidazole scaffold (6 inhibitors, $C 7$ set) and at the $\mathrm{C} 4$ position (4 inhibitors, $C 4$ set), the two substituent sets interacting predominantly with different subpockets of the TbPTR1 protein.

For evaluation of the inhibitor-receptor interaction energies, we used the recently developed $E_{E L, M T P}^{(10)}+E_{D a s}$ approximate model, including only multipole electrostatic and dispersive energy terms [7]. Combining these two terms is reasonable as they both are major long-range contributions to the intermolecular interaction energy. It is noteworthy that, despite the approximate approach, the model does not contain empirical parameters, i.e. it is parametrized only based on the theoretical SAPT results. The model, however, also involves the following approximations: $(i)$ a reduced binding pocket representation that includes only the residues directly interacting with the inhibitors, and (ii) inhibitor-receptor interaction energy terms calculated in a pairwise manner, summing over contributions of each binding pocket residue. This calculation scheme, despite its simplicity, resulted in a good correlation with experimental inhibitory activities for both the $C 7$ and the $C 4$ sets of the TbPTR 1 inhibitors. Furthermore, this computationally efficient model was more accurate in predicting inhibitory activities of TbPTR1 inhibitors than the extra-precision (XP) docking score of Glide (Schrödinger, Inc.). We also found that the replacement of the $E_{\text {Das }}$ function by the D3 correction to dispersionless-DFT could also be considered, since this approximation reflects the correlation energy contribution as accurately as the $E_{\text {Das }}$ energy. Unlike the latter, however, the D3 correction might be dependent on the choice of the functional and further validation is required to assess its range of applicability. Considering both the $C 7$ and $C 4$ sets of inhibitors, we obtained correlations with the experimental data for the $E_{E L, M T P}^{(10)}+E_{D a s}$ model. For the more closed, confined $C 4$ subpocket, the $E_{E L, M T P}^{(10)}+E_{D a s}$ model performed less well but gave results comparable to those obtained for FAAH inhibitors [7]: $R=-0.59$ versus -0.67 , respectively. The lower correlation for the $C 4$ inhibitor set may be due to the low number of compounds with a narrow range of experimental $p \mathrm{~K}_{\mathrm{i}}^{a p p}$ values. We found that $E_{E L, M T P}^{(10)}+E_{\text {Das }}$ is better able to overcome deficiencies of the model of the ligand-receptor complex for the halogenated compound (inhibitor 24) than the interaction energy at the MP2 level of theory. However, the applicability of the $E_{E L, M T P}^{(10)}+E_{D a s}$ model is limited by the omission of the $E_{E X}^{(10)}$ term, as seen for inhibitor 25 whose fragment is slightly too bulky to fit well in the $C 4$ subpocket. Finally, we have shown that the $E_{E L, M T P}^{(10)}+E_{D a s}$ model for the $C 7$ subpocket could be transferred to the $C 4$ subpocket with refitting of the $\beta$ constant term. The model could therefore be applied to the prediction of novel compounds capable of reversible binding to the target enzyme. The observed partial transferability and favorable computational scaling of the $E_{E L, M T P}^{(10)}+E_{\text {Das }}$ model opens possibilities for future applications in lead optimization. The most accurate inhibitory activity predictions can be expected for a set of compounds with similar solvation energy and the binding poses that share a common characteristics. The transferability of the $E_{E L, M T P}^{(10)}+E_{D a s}$ model between different subpockets should be investigated further for larger sets of compounds with more diverse chemical properties.

Acknowledgements The project was financed in part by a statutory activity subsidy No. 0401/0259/16 from the Polish Ministry of Science and Higher Education for the Faculty of Chemistry at the Wrocław University of Technology and Heidelberg Institute for Theoretical Studies (HITS). We thank the BioNaM Project financed from the European Social Fund and Human Capital Operational Programme for the support. We gratefully acknowledge the support of the Klaus Tschira Foundation. This project has received funding from the European Union's Seventh Framework Programme for research, technological development and demonstration under Grant Agreement No. 603240 (NMTrypI - New Medicine for Trypanosomatidic Infections, http://www.nmtrypi.eu/). JPH additionally acknowledges support from the Polish National Science Centre (grant no. 2016/21/D/NZ1/02806) and BIOMS program at the Interdisciplinary Center for Scientific Computing (IWR), Heidelberg University. Calculations were performed at the Wrocław Supercomputer and Networking Center (WCSS) and Heidelberg Institute for Theoretical Studies (HITS).

Open Access This article is distributed under the terms of the Creative Commons Attribution 4.0 International License (http:// creativecommons.org/licenses/by/4.0/), which permits unrestricted use, distribution, and reproduction in any medium, provided you give appropriate credit to the original author(s) and the source, provide a link to the Creative Commons license, and indicate if changes were made.

\section{References}

1. Leach AR, Shoichet BK, Peishoff CE (2006) Prediction of protein-ligand interactions. Docking and scoring: successes and gaps. J Med Chem 49:5851-5855 
2. Doweyko AM (2004) 3D-QSAR illusions. J Comput Aided Mol Des 18:587-596

3. Yilmazer ND, Korth M (2013) Comparison of molecular mechanics, semi-empirical quantum mechanical, and density functional theory methods for scoring protein-ligand interactions. J Phys Chem B 117:8075-8084

4. Carlson HA, Smith RD, Damm-Ganamet KL, Stuckey JA, Ahmed A, Convery MA, Somers DO, Kranz M, Elkins PA, Cui G, Peishoff CE, Lambert MH, Dunbar JB Jr (2016) CSAR 2014: a benchmark exercise using unpublished data from pharma. $\mathrm{J}$ Chem Inf Model 6:1063-1077

5. Dyguda E, Grembecka J, Sokalski WA, Leszczyński J (2005) Origins of the activity of PAL and LAP enzyme inhibitors: towards ab initio binding affinity prediction. J Am Chem Soc 127:1658-1659

6. Grembecka J, Kędzierski P, Sokalski WA, Leszczyński J (2001) Electrostatic models of inhibitory activity. Int J Quantum Chem 83(3-4):180-192

7. Giedroyć-Piasecka W, Dyguda-Kazimierowicz E, Beker W, Mor M, Lodola A, Sokalski WA (2014) Physical nature of fatty acid amide hydrolase interactions with its inhibitors: testing a simple nonempirical scoring model. J Phys Chem B 118(51):14727-14736

8. Lonsdale R, Harvey JN, Mulholland AJ (2010) Inclusion of dispersion effects significantly improves accuracy of calculated reaction barriers for cytochrome $\mathrm{P} 450$ catalyzed reactions. J Phys Chem Lett 1:3232-3237

9. Cornell WD, Cieplak P, Bayly CI, Gould IR, Merz KM, Ferguson DM, Spellmeyer DC, Fox T, Caldwell JW, Kollman PA (1995) A second generation force field for the simulation of proteins, nucleic acids, and organic molecules. J Am Chem Soc 117:5179-5197

10. Kolár M, Berka K, Jurečka P, Hobza P (2010) On the reliability of the AMBER force field and its empirical dispersion contribution for the description of noncovalent complexes. ChemPhysChem 11:2399-2408

11. Zgarbová M, Otyepka M, Šponer J, Hobza P, Jurečka P (2010) Large-scale compensation of errors in pairwise-additive empirical force fields: comparison of AMBER intermolecular terms with rigorous DFT-SAPT calculations. Phys Chem Chem Phys 12:10476-10493

12. Paton RS, Goodman JM (2009) Hydrogen bonding and pi-stacking: how reliable are force fields? A critical evaluation of force field descriptions of nonbonded interactions. J Chem Inf Model 49:944-955

13. Wieczorek R, Haskamp L, Dannenberg JJ (2004) Molecular orbital calculations of water clusters on counterpoise-corrected potential energy surfaces. J Phys Chem A 198:6713-6723

14. Wu Q, Yang WT (2002) Empirical correction to density functional theory for van der waals interactions. J Chem Phys 116:515-524

15. Grimme S (2004) Accurate description of van der Waals complexes by density functional theory including empirical corrections. J Comput Chem 25:1463-1473

16. Korth M (2013) Error estimates for (semi-)empirical dispersion terms and large biomacromolecules. Org Biomol Chem 11:6515-6519

17. Pernal K, Podeszwa R, Patkowski K, Szalewicz K (2009) Dispersionless density functional theory. Phys Rev Lett 103(26):263201

18. Jeziorski B, Moszyński R, Szalewicz K (1994) Perturbation theory approach to intermolecular potential energy surfaces of van der Waals complexes. Chem Rev 94(7):1887-1930

19. Podeszwa R, Pernal K, Patkowski K, Szalewicz K (2010) Extension of the Hartree-Fock plus dispersion method by first-order correlation effects. J Phys Chem Lett 1(2):550-555
20. Spinks D, Ong HB, Mpamhanga CP, Shanks EJ, Robinson DA, Collie IT, Read KD, Frearson JA, Wyatt PG, Brenk R, Fairlamb AH, Gilbert IH (2011) Design, synthesis and biological evaluation of novel inhibitors of Trypanosoma brucei pteridine reductase 1. ChemMedChem 6(2):302-308

21. Nare B, Hardy LW, Beverley SM (1997) The roles of pteridine reductase 1 and dihydrofolate reductase-thymidylate synthase in pteridine metabolism in the protozoan parasite Leishmania major. J Biol Chem 272(21):13883-13891

22. Robello C, Navarro P, Castanys S, Gamarro F (1997) A pteridine reductase gene ptr1 contiguous to a P-glycoprotein confers resistance to antifolates in Trypanosoma cruzi. Mol Biochem Parasitol 90(2):525-535

23. Mpamhanga CP, Spinks D, Tulloch LB, Shanks EJ, Robinson DA, Collie IT, Fairlamb AH, Wyatt PG, Frearson JA, Hunter WN, Gilbert IH, Brenk R (2009) One scaffold, three binding modes: novel and selective pteridine reductase 1 inhibitors derived from fragment hits discovered by virtual screening. J Med Chem 52(14):4454-4465

24. Cavazzuti A, Paglietti G, Hunter WN, Gamarro F, Piras S, Loriga M, Allecca S, Corona P, McLuskey K, Tulloch L, Gibellini F, Ferrari S, Costi MP (2008) Discovery of potent pteridine reductase inhibitors to guide antiparasite drug development. Proc Natl Acad Sci USA 105(5):1448-1453

25. Corona P, Gibellini F, Cavalli A, Saxena P, Carta A, Loriga M, Luciani R, Paglietti G, Guerrieri D, Nerini E, Gupta S, Hannaert V, Michels PAM, Ferrari S, Costi PM (2012) Structure-based selectivity optimization of piperidine-pteridine derivatives as potent Leishmania pteridine reductase inhibitors. J Med Chem 55(19):8318-8329

26. Dawson A, Gibellini F, Sienkiewicz N, Tulloch LB, Fyfe PK, McLuskey K, Fairlamb AH, Hunter WN (2006) Structure and reactivity of Trypanosoma brucei pteridine reductase: inhibition by the archetypal antifolate methotrexate. Mol Microbiol 61(6):1457-1468

27. Sastry GM, Adzhigirey M, Day T, Annabhimoju R, Sherman W (2013) Protein and ligand preparation: parameters, protocols, and influence on virtual screening enrichments. J Comput Aided Mol Des 27:221-234

28. Schrödinger LLC, New York NY (2015) Schrödinger Suite 2015-2. Maestro version 10:2

29. Schrödinger LLC, New York, NY (2015) Schrödinger Suite 2015-2, Protein Preparation Wizard, Epik version 3.2, Impact version 6.7, Prime version 4.0

30. Banks J, Beard H, Cao Y, Cho A, Damm W, Farid R, Felts A, Halgren T, Mainz D, Maple J, Murphy R, Philipp D, Repasky M, Zhang L, Berne B, Friesner R, Gallicchio E, Levy R (2005) Integrated modeling program, applied chemical theory (IMPACT). J Comp Chem 26:1752-1780

31. Li H, Robertson A, Jensen J (2005) Very fast empirical prediction and interpretation of protein $\mathrm{pKa}$ values. Proteins 61:704-721

32. Bas D, Rogers D, Jensen J (2008) Very fast prediction and rationalization of $\mathrm{pKa}$ values for protein-ligand complexes. Proteins 73:765-783

33. Olsson M, Søndergard C, Rostkowski M, Jensen J (2011) PROPKA3: consistent treatment of internal and surface residues in empirical pka predictions. J Chem Theory Comput 7:525-537

34. Søndergard C, Olsson M, Rostkowski M, Jensen J (2011) Improved treatment of ligands and coupling effects in empirical calculation and rationalization of $\mathrm{pKa}$ values. J Chem Theory Comput 7:2284-2295

35. Friesner RA, Banks JL, Murphy RB, Halgren TA, Klicic JJ, Mainz DT, Repasky MP, Knoll EH, Shelley M, Perry JK, Shaw DE, Francis P, Shenkin PS (2004) Glide: a new approach for 
rapid, accurate docking and scoring. 1. method and assessment of docking accuracy. J Med Chem 47(7):1739-1749

36. Friesner RA, Murphy RB, Repasky MP, Frye LL, Greenwood JR, Halgren TA, Sanschagrin PC, Mainz DT (2006) Extra precision glide: docking and scoring incorporating a model of hydrophobic enclosure for protein-ligand complexes. J Med Chem 49(21):6177-6196

37. Gourley D, Leonard ASG, Luba J, Hardy L, Beverley S, Hunter W (2001) Pteridine reductase mechanism correlates pterin metabolism with drug resistance in trypanosomatid parasites. Nature Struct Biol 8(6):521-525

38. Sokalski WA, Roszak S, Pecul K (1988) An efficient procedure for decomposition of the scf interaction energy into components with reduced basis set dependence. Chem Phys Lett 153:153-159

39. Góra R, Sokalski WA, Leszczyński J, Pett V (2005) The nature of interactions in the ionic crystal of 3-pentenenitrile, 2-nitro-5oxo, ion(-1) sodium. J Phys Chem B 109:2027-2033

40. Sokalski WA, Sawaryn A (1987) Correlated molecular and cumulative atomic multipole moments. J Chem Phys 87:526-534

41. Schmidt MW, Baldridge KK, Boatz JA, Elbert ST, Gordon MS, Jensen JH, Koseki S, Matsunaga N, Nguyen KA, Su SJ, Windus TL, Dupuis M, Montgomery JA (1993) General atomic and molecular electronic structure system. J Comput Chem 14(11):1347-1363

42. Krishnan R, Binkley JS, Seeger R, Pople JA (1980) Selfconsistent molecular orbital methods. XX. A basis set for correlated wave functions. J Chem Phys 72(1):650-654

43. McLean AD, Chandler GS (1980) Contracted gaussian basis sets for molecular calculations. I. Second row atoms, $Z=11-18$. J Chem Phys 72(10):5639-5648

44. Wachters AJH (1970) Gaussian basis set for molecular wavefunctions containing third-row atoms. J Chem Phys 52:1033-1036

45. Hay PJ (1977) Gaussian basis sets for molecular calculationsrepresentation of 3D orbitals in transition-metal atoms. J Chem Phys 66:4377-4384

46. Boys S, Bernardi F (1970) The calculation of small molecular interactions by the differences of separate total energies. Some procedures with reduced errors. Mol Phys 19:553-566

47. Sokalski WA, Poirier RA (1983) Cumulative atomic multipole representation of the molecular charge distribution and its basis set dependence. Chem Phys Lett 98(1):86-92

48. Grimme S, Antony J, Ehrlich S, Krieg H (2010) A consistent and accurate $a b$ initio parametrization of density functional dispersion correction (DFT-D) for the 94 elements H-Pu. J Chem Phys 132(15): 154104

49. Grimme S, Ehrlich S, Goerigk L (2011) Effect of the damping function in dispersion corrected density functional theory. J Comput Chem 32(7):1456-1465

50. Perdew JP, Burke K, Ernzerhof M (1996) Generalized gradient approximation made simple. Phys Rev Lett 77(18):3865-3868

51. Adamo C, Barone V (1999) Toward reliable density functional methods without adjustable parameters: the PBE0 model. J Chem Phys 110(13):6158-6170

52. Becke AD (1993) Density-functional thermochemistry. III. The role of exact exchange. J Chem Phys 98(7):5648-5652

53. Tao JM, Perdew JP, Staroverov VN, Scuseria GE (2003) Climbing the density functional ladder: nonempirical meta-generalized gradient approximation designed for molecules and solids. Phys Rev Lett 91(14):146401

54. Langner K, Beker W, Sokalski W (2012) Robust predictive power of the electrostatic term at shortened intermolecular distances. J Phys Chem Lett 3:2785-2789

55. Schrödinger LLC, New York NY (2015) Schrödinger Suite 2015-2. Glide version 6:7

56. Eldridge MD, Murray CW, Auton TR, Paolini GV, Mee RP (1997) Empirical scoring functions: I. The development of a fast empirical scoring function to estimate the binding affinity of ligands in receptor complexes. J Comput Aided Mol Des 11(5):425-445

57. Berg JM, Tymoczko JL, Stryer L (2012) Biochemistry, 7th edn. W.H. Freeman, Basingstoke

58. Grzywa R, Dyguda-Kazimierowicz E, Sieńczyk M, Feliks M, Sokalski WA, Oleksyszyn J (2007) The molecular basis of urokinase inhibition: from the nonempirical analysis of intermolecular interactions to the prediction of binding affinity. J Mol Model 13(6-7):677-683

59. Klebe G (2006) Virtual ligand screening: strategies, perspectives and limitations. Drug Discov Today 11:580-594

60. Plewczyński D, Łaźniewski M, Augustyniak R, Ginalski K (2011) Can we trust docking results? Evaluation of seven commonly used programs on PDBbind database. J Comput Chem 32:742-755

61. Wilcken R, Zimmermann MO, Lange A, Joerger AC, Boeckler FM (2013) Principles and applications of halogen bonding in medicinal chemistry and chemical biology. J Med Chem 56(4):1363-1388 